\section{A millifluidic-based 3D-plat- form as a useful tool for the efficacy of glaucoma therapeutic strategies}

\author{
Anna M. Bassi,1,2 Sara Tirendi,1,2 \\ Sonia Scarfi, 2,3 Mario Passalacqua, 1,2 \\ Vanessa Almonti, 1,2 Stefania Vernazza1 \\ 1 Department of Experimental Medicine \\ (DIMES), University of Genoa; 2Inter- \\ University Center for the Promotion of \\ the 3Rs Principles in Teaching \& \\ Research (Centro 3R), Pisa; \\ 3Department of Earth, Environment and \\ Life Sciences (DISTAV), University of \\ Genoa, Italy
}

\begin{abstract}
The aim of this present study is to investigate the role of damaged Trabecular Meshwork (TM) in triggering neuron-like cell apoptosis. Preliminary results showed that stressed TM releases harmful signals to neuron-like cells, suggesting its pivotal role in glaucoma cascade.
\end{abstract}

\section{Introduction}

Glaucoma is characterized by optic nerve damage and the slow progressive death of Retinal Ganglion Cells (RGCs). To date, although the main clinical approach is the management of Intraocular Pressure (IOP) by surgery or drugs, these have not turned out to be successful in preventing vision loss. ${ }^{1}$ Indeed, RGC death is also driven by different converging molecular pathways, engaged in additional damage more or less closely connected to IOP elevation, which are able to trigger or exacerbate the glaucomatous cascade. ${ }^{2}$ For example, defects in the Trabecular Meshwork (TM), one of the main tissues involved in the conventional outflow pathway, are responsible for IOP elevation in high tension glaucoma. ${ }^{3}$ Therefore, given that, in the anterior segment of the eye, the TM is the most sensitive tissue to Oxidative Stress (OS), we aimed to verify whether the TM plays an active role in triggering $\mathrm{RGC}$ death. 4

Thus, we used a millifluidic-human based 3D-platform in vitro, recently set up by us, ${ }^{5-7}$ to analyze the potential role of oxidative stressed-TM cells in affecting the survival of neural-like cells, as a model of RGCs.

\section{Materials and Methods}

\section{Cell Cultures}

Human Trabecular Meshwork Cells (HTMC) and Trabecular Meshwork Growth Medium (TMGM) were obtained by Cell Application INC (San Diego, CA, USA); human neuroblastoma cells (SH-SY5Y) (American Type Culture Collection; Gaithersburg, MD, USA) were used as a model of retinal cells, since they can differentiate towards the neuronal-like phenotype (N-SH-SY5Y) by being grown in RPMI medium supplemented with $10 \mu \mathrm{M}$ retinoic acid (RA). ${ }^{8}$ Both $3 \mathrm{D}$ cell cultures and the dynamic culture system were carried out as previously described.5,7 Briefly, 3D HTMC and N-SH-SY5Y cultures embedded in Matrigel ${ }^{\circledR}$ Matrix, were set up under dynamic conditions by using millifluidic bioreactors connected to a peristaltic pump (Live Box 1 and Live Flow, IV-Tech srl, Massarosa, LU, Italy) with a constant flow rate $(70 \mathrm{~mL} / \mathrm{min})$.

\section{Experimental conditions}

Direct effects of prolonged OS were investigated by treating 3D HTMCs up to $72 \mathrm{~h}$, by daily exposure to $500 \mu \mathrm{M} \mathrm{H}_{2} \mathrm{O}_{2}$ for $2 \mathrm{~h}$, with 22-hour recovery phases in between (Figure 1).

Next, the indirect effects of OS were analyzed on differentiated 3D N-SHSY 5 Ys using $50 \%$ of RPMI, supplemented with RA, and $50 \%$ of conditioned medium derived from OS-treated HTMC up to $72 \mathrm{~h}$.

\section{Viability Assay}

Alamar Blue (AB) assay from Invitrogen ${ }^{\mathrm{TM}}$ (Life Technologies, Carlsbad, CA, USA) was performed according to manufacturer's instructions, to analyze the metabolic activity daily of both the 3DHTMC and N-SH-SY5Y cells. The AB assay was carried out a) on HTMCs to analyze the selective toxicity induced by prolonged $\mathrm{H}_{2} \mathrm{O}_{2}$ exposure; and b) on N-SHSY5Y cells to verify the role of stressedHTMCs in triggering any distress on neural-like cells during their growth in the presence of conditioned medium derived from OS-treated HTMCs.

\section{Human apoptosis array}

Analysis of the apoptosis proteomic profile of 3D-HTMC and 3D-N-SH-SY5Y after experimental procedures was carried out by commercial Human Apoptosis Antibody Array C1 (RayBio ${ }^{\circledR}$; Norcross, GA, USA), a semi-quantitative detector of human proteins. The intensity of protein array signals was analyzed using a BIORAD Geldoc 2000 and each protein spot was normalized against Positive Control
Correspondence:Anna M. Bassi, Department of Experimental Medicine (DIMES), University of Genoa, Italy; Inter-University Center for the Promotion of the 3Rs Principles in Teaching \& Research (Centro 3R), Italy. E-mail: anna.maria.bassi@unige.it

Key words: Glaucoma; in vitro model; trabecular meshwork.

Acknowledgments: We would like to express our gratitude to IVTech srl (Massarosa, LU, Italy) for their technical support.

Disclosures: Authors have nothing to disclose.

Conference presentation: This paper was presented at the Third Centro 3R Annual Meeting - L'era delle 3R: modelli in silico, in vitro e in vivo per promuovere la ricerca traslazionale 30 September - 1 October 2021, Evento online organizzato dal Politecnico di Torino.

Received for publication: 9 July 2021.

Accepted for publication: 7 September 2021.

This work is licensed under a Creative Commons Attribution NonCommercial 4.0 License (CC BY-NC 4.0).

CC Copyright: the Author(s), 2021

Licensee PAGEPress, Italy

Biomedical Science and Engineering 2021; 4(s1):135 doi:10.4081/bse.2021.135

Spots printed on each membrane. The data analysis was conducted following the Protocol instructions of the Human Apoptosis Array C1.

\section{Statistical analysis}

GraphPad Prism 7.0 (GraphPad. Software, San Diego, CA, USA) was used for the statistical analysis. For the intergroup comparison of viability assay, we used the two-way analysis of variance (ANOVA) for single comparisons.

\section{Results}

As shown in Figure 2, the prolonged exposure to $500 \mu \mathrm{M} \mathrm{H}_{2} \mathrm{O}_{2}$, as an oxidative stressor, affected the healthy state of the HTMCs already after $24 \mathrm{~h}$, whilst the N-SHSY5Ys, during their growth in the presence of $50 \%$ conditioned medium derived from stressed-HTMCs, displayed a reduction of their metabolic state, only starting from $48 \mathrm{~h}$ of experimental treatment. Moreover, extrapolated data from the heat map analysis evidenced an increase in apoptotic protein expressions in OS-treated HTMCs, mainly at $48 \mathrm{hrs}(\mathrm{p}<0.01)$. Conversely, the $\mathrm{N}-\mathrm{SH}-\mathrm{SY} 5 \mathrm{Ys}$ increased the levels of pro- 


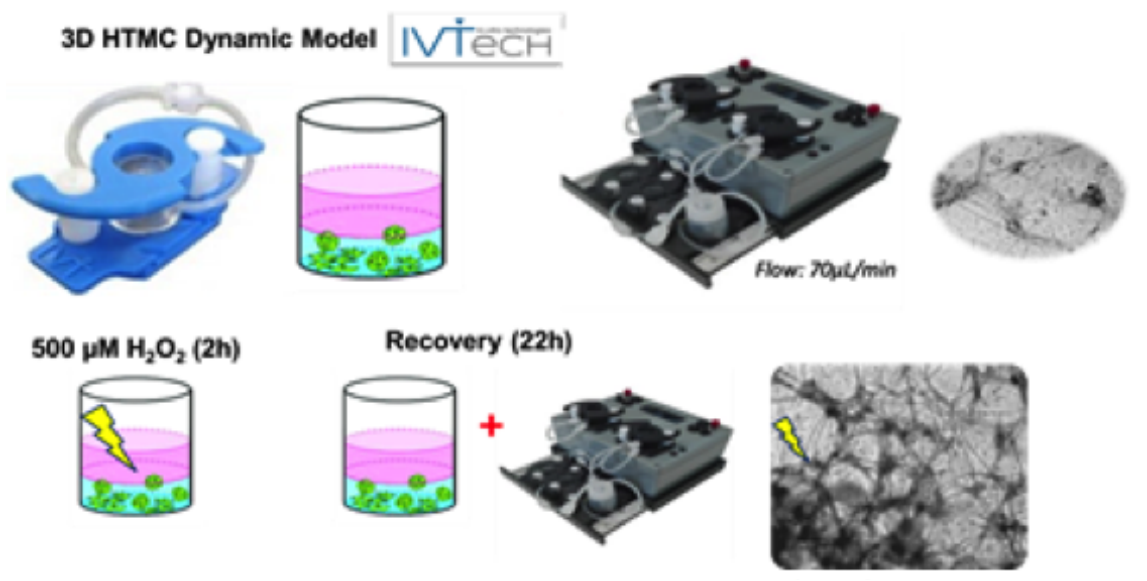

Figure 1. Human 3D HTMC - platform experimental model exposed to oxidative stress conditions. Representative images (on right) at contrast phase microscopy during OStreatment.

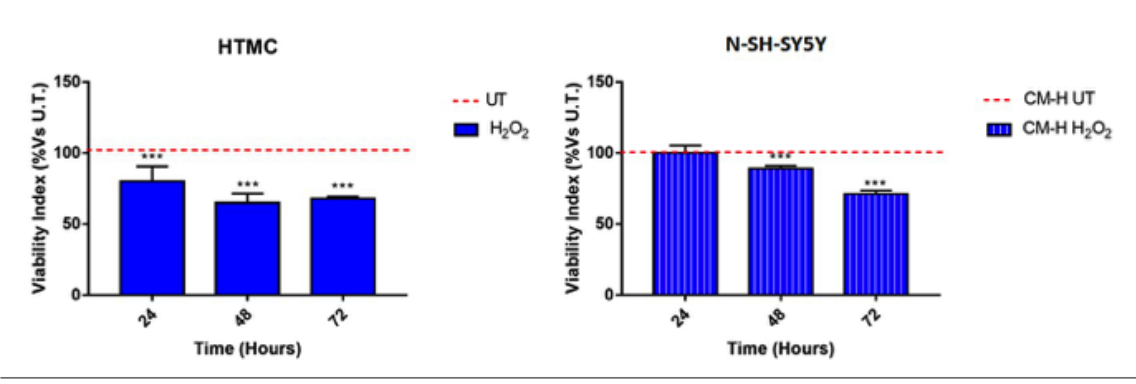

Figure 2. Viability index. Metabolic state of HTMCs and N-SH-SY5Ys during experimental conditions was analyzed by Alamar blue assay. Dotted red line represents viability index of untreated cells. Data are expressed as \% vs. untreated cells and each value represents the mean \pm SD of 3 independent experiments running in triplicate. UT: untreated cultures; CM-H: conditioned medium derived from HTMC cultures.

apoptotic proteins $(\mathrm{p}<0.01)$ only after $72 \mathrm{~h}$ of the above-mentioned experimental conditions.

\section{Discussion and Conclusions}

These preliminary results demonstrate that HTMCs, during prolonged exposure to OS are able to release biomolecules which act as pro-apoptotic signals for N-SH-
SY5Ys. Therefore, it can be assumed that in glaucoma, RGC cell death could also be promoted by a damaged TM.

\section{References}

1. Quaranta L, Micheletti E, Carassa R, et al. Efficacy and safety of PreserFlo ${ }^{\circledR}$ MicroShunt after a failed trabeculectomy in eyes with primary open-angle glaucoma: A retrospective study. Adv Ther 2021;38:4403-12.

2. Vernazza S, Tirendi S, Bassi AM, et al. Neuroinflammation in Primary OpenAngle Glaucoma. J Clin Med 2020;9:3172.

3. Saccà SC, Cutolo CA, Rossi T. Glaucoma: An Overview. in Preedy V, Watson RR (eds). Handbook of Nutrition, Diet, and the Eye. Elsevier; Amsterdam, The Netherlands: 2019, pp. $167-187$

4. Saccà SC, Gandolfi S, Bagnis A, et al. From DNA damage to functional changes of the trabecular meshwork in aging and glaucoma. Ageing Res Rev 2016;29:26-41.

5. Vernazza S, Tirendi S, Scarfî S, et al. 2Dand 3D-cultures of human trabecular meshwork cells: A preliminary assessment of an in vitro model for glaucoma study. PLoS One 2019;14:e221942.

6. Saccà SC, Izzotti A, Vernazza S, et al. Can Polyphenols in Eye Drops Be Useful for Trabecular Protection from Oxidative Damage? J Clin Med 2020;9:3584.

7. Saccà SC, Tirendi S, Scarfî S, et al. An advanced in vitro model to assess glaucoma onset. ALTEX 2020;37:265-74.

8. Nitti M, Furfaro AL, Cevasco C, et al. PKC delta and NADPH oxidase in retinoic acid-induced neuroblastoma cell differentiation. Cell Signal 2010;22:828-35. 